\title{
Rare complications during deep brain stimulation surgery for Parkinson's disease
}

\section{Ponniah Vanamoorthy, Amit Tomar', Hemanshu Prabhakar', Girija Prasad Rath ${ }^{1}$}

Deep brain stimulation (DBS) of subthalamic nucleus and globus pallidus is increasingly being used for treatment of Parkinson's disease (PD) and other movement disorders. The procedure involves stereotactic placement of an implantable electrode with four active contacts within the target following which the external cables of the electrodes are tunneled and connected to a pulse generator, which is implanted externally. A wide array of complications can occur during this procedure from initial patient selection to years and decades after surgery. ${ }^{[1,2]}$ Complications focused on literatures are related to the device, procedure, electrical stimulation and spread of current. ${ }^{[3,4]}$ Some of these include intracerebral haematoma, ischaemic stroke, seizures, lead migration, erosion and breakage, dyskinesias, dysarthria, mood changes, Horner's syndrome, etc., Of particular concern to the anaesthesiologists are the perioperative complications, some of which in our experience were unusual.

We analysed all the patients undergoing bilateral simultaneous subthalamic nucleus DBS surgery over a period of 3 years. The procedure involved fixing of metal stereotactic frame under local anaesthesia, location of the target with magnetic resonance imaging (MRI) and computer assistance, stereotactic placement of stimulating/recording electrode in the target, and intraoperative electrophysiological monitoring to confirm location of the tip of stimulating electrode, and replacement of the stimulating electrode by implantable multicontact electrode. The same procedure is repeated on the opposite side. Then, the external cables of the electrodes are tunneled below the scalp and behind the ear, and connected to an implanted pulse generator which is commonly placed in a subcutaneous pocket below the clavicle. The surgery was performed in one setting, in all the patients. Continuous monitoring of ECG, pulse oximetry, non-invasive blood pressure and skin temperature was commenced. Besides routine intraoperative monitoring, bispectral index (BIS) monitoring was also done. Electrodes were placed under monitored anaesthesia care (MAC) with minimal (Target BIS 80) or no sedation and supplemental oxygen was given to all the patients via nasal prongs $(2 \mathrm{l} / \mathrm{min})$. Implantation of pulse generator was done under general anaesthesia with a standardised anaesthetic technique. All the patients were induced with fentanyl $2 \mu \mathrm{g} / \mathrm{kg}$ and thiopentone 4-5 mg/ kg until loss of eyelash reflex; rocuronium $1 \mathrm{mg} / \mathrm{kg}$ was administered to facilitate endotracheal intubation. Anaesthesia was maintained with isoflurane in nitrous oxide and $40 \% \mathrm{O}_{2}$, and incremental doses of fentanyl $1 \mu \mathrm{g} / \mathrm{kg}$ and vecuronium $0.03 \mathrm{mg} / \mathrm{kg}$. At the end of the procedure, neuromuscular blockade was reversed with neostigmine $0.05 \mathrm{mg} / \mathrm{kg}$ and glycopyrrolate $0.01 \mathrm{mg} / \mathrm{kg}$ and trachea was extubated.

Departments of Neuroanaesthesiology, Global Health City, Chennai, ${ }^{1}$ Neuroanaesthesiology, All India Institute of Medical Sciences, New Delhi, India

Address for correspondence:

Dr. Girija Prasad Rath, Department of Neuroanaesthesiology, Neurosciences Centre, All India Institute of Medical Sciences, New Delhi - 110029 , India. E-mail: girijarath@yahoo.co.in 
Postoperatively, all the patients were observed for $24 \mathrm{~h}$ in the neurosurgical ICU.

Nineteen patients ( 5 females and 14 males) with a mean age of 49.7 years (range 15-67 yr) who underwent bilateral simultaneous subthalamic nucleus DBS surgery over a period from 2007 to 2010 were analysed for the study. Three patients developed unexpected complications in the form of supraventricular arrhythmias, tension pneumocephalus and subdural haematoma. Although subdural haematoma following DBS has been described in literature, arrhythmias and tension pneumocephalus ${ }^{[5]}$ are rare and hence, these two cases are described in detail.

The first patient was a 67-yr-old male with a remarkable medical history for diabetes mellitus and hypertension controlled on oral hypoglycemic and antihypertensive medications, respectively. Stimulation of the central and anterior leads of right subthalamic nucleus caused appearance of supraventricular ectopics [Figure 1a and b], albeit, with unchanged haemodynamics. No neurological deterioration was noted during the event. The arrhythmias appeared each time the electrode was stimulated. Readjustment of the electrode and further procedure was uneventful.

The second patient was a 63-yr-old male with significant medical history for coronary artery disease for 6 years. A two-dimensional echocardiogram revealed concentric left ventricular hypertrophy with mild aortic regurgitation and a left ventricular ejection fraction of $45 \%$. The electrode placement lasted for 5 hours during which the BIS value was maintained at a normal range except at the end when the patient become drowsy (BIS 70-80) believed to be because of sedative agents. However, after placement of pulse generator under anaesthesia, the patient remained drowsy with a BIS value of 65-70. He was shifted to the ICU for close observation and follow-up. Due to persistent drowsiness even after 1 hour in the ICU, a computed tomographic (CT) scan was done which revealed tension pneumocephalus [Figure 2]. The BIS was continuously monitored in the postoperative period. For the initial $10 \mathrm{~h}$ after surgery, BIS value remained in the range of 65-80; later it was increased coinciding with improved sensorium of the patient. The patient was shifted to postoperative ward after $18 \mathrm{~h}$ with intact sensorium and a BIS value of 98 . A CT scan done $24 \mathrm{~h}$ later showed a complete resolution of pneumocephalus.

DBS involves stereotactic placement of an electrode in the target which is connected to a pulse generator. ${ }^{[6]}$ Various adverse effects of DBS related to the surgical

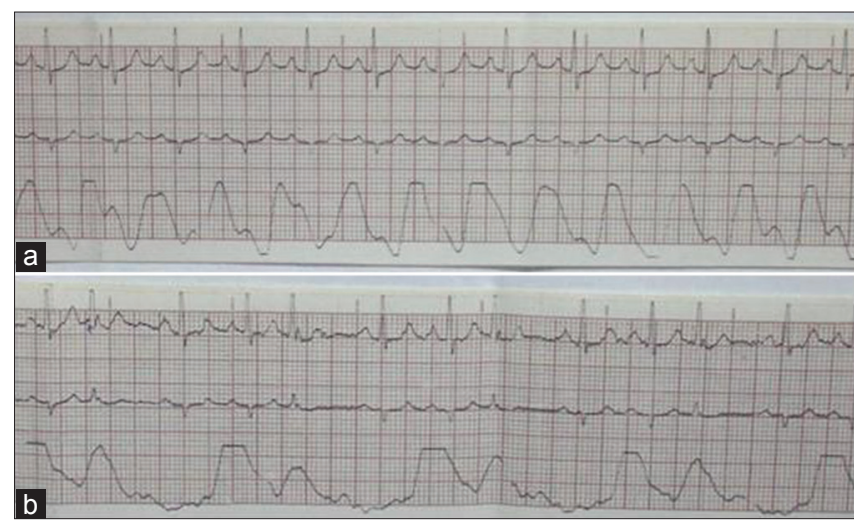

Figure 1: (a) ECG in Lead II showing a normal sinus rhythm prior to stimulation of electrodes placed in the subthalamic nucleus; (b) ECG in Lead II showing supraventricular ectopics during stimulation of electrodes placed in the subthalamic nucleus

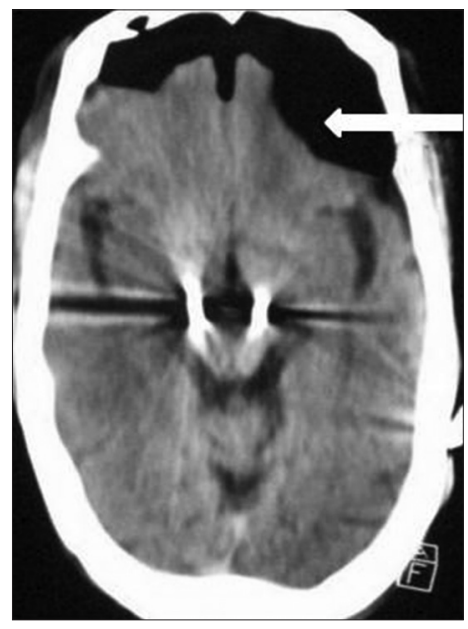

Figure 2: Computed tomographic scan showing accumulated air in the frontal region (bold white arrow) causing tension pneumocephalus

procedure, the implanted device, and electrical stimulation of areas adjacent to the target have been described in literature. ${ }^{[7]}$ Of particular concern to the anaesthesiologists are the perioperative complications. However, as we noticed with increase in number of such procedures, unexpected complications can also occur in the form of arrhythmias and tension pneumocephalus.

Neuro-cardiac links have been shown to produce arrhythmia both experimentally and clinically. Specific examples include stroke, epilepsy and environmental stress. It is hypothesised that the individual with a diseased heart has greater likelihood of experiencing cardiac arrhythmias and sudden cardiac death when the neuro cardiac axis is activated. ${ }^{[8]}$ Electrical stimulation of numerous areas in the midbrain reticular formation and in the posterior hypothalamus of the cat increased blood pressure and caused a 
variety of ECG changes including sinus tachycardia, ventricular premature contractions, bigeminal rhythm, atrio-ventricular dissociation and ventricular tachycardia. ${ }^{[9]}$ Microstimulation of the rat posterior insular cortex resulted in escape rhythms, ventricular ectopics and ultimately death in asystole. ${ }^{[10,11]}$ In patients who underwent intraoperative insular stimulation prior to temporal lobectomy for seizure control, bradycardia and depressor responses were more frequently produced than tachycardia and pressor response on stimulation of the left cortex. ${ }^{[12]}$ Although experimental models indicate that insular lesions may be arrhythmogenic and insular cortex stimulation produces lethal cardiac arrhythmias, it is unknown whether stimulation of subthalamic nucleus can produce arrhythmias. The supraventricular arrhythmias that occurred during subthalamic nucleus stimulation needs further studies.

Pneumocephalus has been reported to occur after any craniotomy procedure. Generally, it is asymptomatic but occasionally high pressure may build up in the air cavity with the development of tension pneumocephalus. Although tension pneumocephalus is more common after posterior fossa or cervical spine surgery in sitting position, it is rare following supine position. ${ }^{[13]}$ During DBS surgery there occurs a slow but continuous egress of CSF from the cranial burr holes. Due to the prolonged duration of surgery, the loss of CSF may become significant over a period of time. This space created by the CSF loss may be filled with air causing pneumocephalus. This seems to be the likely cause of development of pneumocephalus, in our case. It is also likely that during closure of duramater subdural injection of saline to fill such space was insufficient. The use of nitrous oxide in general anaesthesia could have further expanded the collected air resulting in development of tension pneumocephalus manifesting as persistent drowsiness with a low BIS value in the postoperative period. It is probable that over a period of time the accumulated air got reabsorbed resulting in improved BIS values with simultaneous improvement in neurological status.

To conclude, we report our experience with adverse effects in deep brain stimulation surgery for PD. With the increase in number of such procedures, unexpected complications may be anticipated.

\section{REFERENCES}

1. Hariz MI. Complications of deep brain stimulation surgery. Mov Disord 2002;17:S162-6.

2. Venkatraghavan L, Manninen P, Mak P, Lukitto K, Hodaie M, Lozano A. Anesthesia for functional neurosurgery: Review of complications. J Neurosurg Anesthesiol 2006;18:64-7.

3. Beric A, Kelly PJ, Rezai A, Sterio D, Mogilner A, Zonenshayn M, Kopell B. Complications of deep brain stimulation surgery. Stereotact Funct Neurosurg 2001;77:73-8.

4. Terao T, Takahashi H, Yokochi F, Taniguchi M, Okiyama R, Hamada I. Hemorrhagic complications of stereotactic surgery in patients with movement disorders. J Neurosurg 2003;98:1241-6.

5. Jain V, Prabhakar H, Rath GP, Sharma D. Tension pneumocephalus following deep brain stimulation surgery with bispectral index monitoring. Eur J Anaesthesiol 2007;24:203-4.

6. Thompson T, Lunsford LD, Kondziolka D. Technical considerations in movement disorder surgery: Frames, imaging and intraoperative monitoring. Prog Neurol Surg 2000;15:91-102.

7. Valldeoriola F, Pilleri M, Tolosa E, Molinuevo JL, Rumià J, Ferrer E. Bilateral subthalamic stimulation monotherapy in advanced Parkinson's disease: Long term follow-up of patients. Mov Disord 2002;17:125-32.

8. Davis AM, Natelson BH. Brain heart interactions. The neurocardiology of arrhythmia and sudden cardiac death. Tex Heart Inst J 1993;20:158-69.

9. Manning JW, Cotten MD. Mechanisms of cardiac arrhythmias induced by diencephalic stimulation. Am J Physiol 1962;203:1120-4.

10. Oppenheimer SM, Cechetto DF, Hachinski VC. Cerebrogenic cardiac arrhythmias. Cerebral electrocardiographic influences and their role in sudden death. Arch Neurol 1990;47:513-9.

11. Oppenheimer SM, Wilson JX, Guiraudon C, Cechetto DF. Insular cortex stimulation produces lethal cardiac arrhythmias: A mechanism of sudden death? Brain Res 1991;550:115-21.

12. Oppenheimer SM, Gelb A, Girvin JP, Hachinski VC. Cardiovascular effects of human insular cortex stimulation. Neurology 1992;42:1727-32.

13. Prabhakar H, Bithal PK, Garg A. Tension pneumocephalus after craniotomy in supine position. J Neurosurg Anesthesiol 2003; $15: 278-81$.

\begin{tabular}{|l|l|}
\hline \multicolumn{2}{|c|}{ Access this article online } \\
\hline Quick Response Code: & Website: \\
\hline & www.jnaccjournal.org \\
\cline { 2 - 2 } & \\
\hline
\end{tabular}

